Article

\title{
shRNA-Mediated XRCC2 Gene Knockdown Efficiently Sensitizes Colon Tumor Cells to X-ray Irradiation in Vitro and in Vivo
}

Qin Wang ${ }^{1}$, Yan Wang ${ }^{1}$, Liqing Du ${ }^{1}$, Chang Xu ${ }^{1}$, Yuanming Sun ${ }^{1}$, Bing Yang ${ }^{2}$, Zhijuan Sun ${ }^{1}$, Yue Fu ${ }^{1}$, Lu Cai ${ }^{3}$, Saijun Fan ${ }^{1}$, Feiyue Fan ${ }^{1, *}$ and Qiang Liu ${ }^{1, *}$

1 Tianjin Key Lab of Molecular Nuclear Medicine, Institute of Radiation Medicine of Chinese, Academy of Medical Science and Peking Union Medical College, Tianjin 300192, China;

E-Mails: wangqin@irm-cams.ac.cn (Q.W.); wangyan@irm-cams.ac.cn (Y.W.);

dlq@irm-cams.ac.cn (L.D.); xuchang@irm-cams.ac.cn (C.X.);

sunyuanming@irm-cams.ac.cn (Y.S.); sunzj@irm-cams.ac.cn (Z.S.);

wddr0710@gmail.com (Y.F.); fansaijun@irm-cams.ac.cn (S.F.)

2 Department of Cell Biology, Tianjin Medical University, Tianjin 300070, China;

E-Mail: yangbingtj@aol.com

3 Department of Pediatrics of University of Louisville, Louisville, KY 40202, USA;

E-Mail: lu.cai@louisville.edu

* Authors to whom correspondence should be addressed; E-Mails: fanfeiyue@irm-cams.ac.cn (F.F.); liuqiang@irm-cams.ac.cn (Q.L.); Tel.: +86-22-8568-0279 (Q.L.); Fax: +86-22-8568-3033 (Q.L.).

Received: 18 November 2013; in revised form: 30 December 2013 / Accepted: 16 January 2014 / Published: 29 January 2014

Abstract: Colon cancer is one of the most common tumors of the digestive tract. Resistance to ionizing radiation (IR) decreased therapeutic efficiency in these patients' radiotherapy. $\mathrm{XRCC} 2$ is the key protein of DNA homologous recombination repair, and its high expression is associated with enhanced resistance to DNA damage induced by IR. Here, we investigated the effect of XRCC2 silencing on colon tumor cells' growth and sensitivity to X-radiation in vitro and in vivo. Colon tumor cells (T84 cell line) were cultivated in vitro and tumors originated from the cell line were propagated as xenografts in nude mice. The suppression of $X R C C 2$ expression was achieved by using vector-based short hairpin RNA (shRNA) in T84 cells. We found that the knockdown of XRCC2 expression effectively decreased T84 cellular proliferation and colony formation, and led to cell apoptosis and cell cycle arrested in G2/M phase induced by X-radiation in vitro. In addition, tumor xenograft studies suggested that XRCC2 silencing inhibited tumorigenicity 
after radiation treatment in vivo. Our data suggest that the suppression of XRCC2 expression rendered colon tumor cells more sensitive to radiation therapy in vitro and in vivo, implying $X R C C 2$ as a promising therapeutic target for the treatment of radioresistant human colon cancer.

Keywords: XRCC2; RNA interference; colon cancer; ionizing radiation; radiosensitivity

\section{Introduction}

Colorectal cancer, including colon and rectal cancer, is one of the most common tumors of the digestive tract. It is the third most commonly diagnosed cancer in males and the second in females, with over 1.2 million new cancer cases and 608,700 deaths estimated to have occurred in 2008 [1,2]. The best therapy for colon cancer is through surgical excision after early diagnosis, which can be combined with radiotherapy, chemotherapy, and immunotherapy. Radiotherapy before surgery makes tumor volume shrink and improves the removal rate. Radiotherapy after surgery kills remaining tumor cells. Radiotherapy alone is suitable for colon cancer patients in advanced stage, which has the effect of stopping bleeding and pain. Collectively, radiation plays an important role in colon cancer's therapy. However, colon cancer is not sensitive to radiotherapy, prompting to the search of novel strategies to enhance radiosensitivity of colon cancer for reducing cancer recurrence after surgery and improving survival of patients.

The inactivation of tumor suppressor genes, activation of oncogenes or various repair genetic defects in DNA replication is closely associated with colorectal cancer incidence [3]. The double-strand break (DSB) is the main DNA lesion induced by ionizing radiation (IR). Unrepaired DSB or misrepaired DSB may lead to cell death or mutation or carcinogenesis. The two methods of repairing DSB include homologous recombination (HR) and non-homologous end-joining (NHEJ). $\mathrm{HR}$, error-free repair pathway, is an evolutionarily conserved mechanism for the repair DSB without inducing base substitutions, deletions, or insertions. In mammalian cells, repair of DSB via HR is dependent on the RecA/Rad51 family.

$\mathrm{X}$-ray repair cross complementing gene family $(X R C C 1-X R C C 11)$ plays an important role in repairing DNA damage induced by IR. XRCC2 gene, a member of the family, first separated from hamster cells line Irsl, is closely associated with HR. XRCC2 locates on human chromosome 7q36.1, cDNA 1,580 bp, encodes 280 amino acid, which is a key component of HR repair proteins RecA/Rad51family [4]. XRCC2 recruits Rad51 to the broken DNA ends. Then, the complex, formed by XRCC2, Rad51B, Rad51C, and Rad51D, ligases with single-stranded DNA and DSB, which promotes Rad51 to identify location of DSB and assembly Rad51-DNA filaments. It helps to maintain genomic stability [5] and resist various cytotoxic factors (such as IR) damage to DNA [6]. A report found that XRCC2 gene deletion caused the formation defects of the core protein RAD51, which resulted in lack of effective repair of DNA damage and increased the spontaneous chromosomal aberrations and chromosomal abnormalities separation [7]. Therefore, XRCC2 abnormal expression can lead to genomic instability and promote tumor development and progression. 
A recent study showed that XRCC2-deficient cells were defective in HR repair function in response to DSB as compared with the parent cell lines [4]. Consequently, the sensitivity to IR is significantly increased in XRCC2-deficient cells than in normal cells [8]. An investigation suggested that the abnormal upregulation of $X R C C 2$ gene expression rendered lung cancer cells' resistance to DNA damage induced by radiation, which resulted in tumors' resistance to radiotherapy [9]. Thus, we propose that the inhibiting of $X R C C 2$ expression of tumor cells may enhance their radiosensitivity.

In recent years, studies on XRCC 2 focuses on HR repair mechanisms and the relationship between $X R C C 2$ gene polymorphism and tumor susceptibility [10-14], such as the relationship between XRCC2 R188H polymorphism and breast cancer, thyroid cancer, ovarian cancer, etc. Reports on XRCC2 associated with colorectal cancer only deal with XRCC2 polymorphism [15-19]. However, there are no studies on $X R C C 2$ expression in colon cancer and its association with sensitivity to IR. Until now, it is not yet known whether the level of $X R C C 2$ expression can affect the sensitivity of radiotherapy for colon cancer and whether $X R C C 2$ can predict the efficacy of colon cancer radiotherapy. Thus, we adopted shRNA-mediated $X R C C 2$ silencing strategy to investigate the effect of $X R C C 2$ silencing on cell growth and sensitization to $\mathrm{X}$-radiation in colon cancer in vitro and in vivo.

\section{Results}

\subsection{Expression of XRCC2 in Various Tumor Cell Lines and Normal Cell Line}

To clarify whether $X R C C 2$ expression is abnormal in human colon cancer, we analyzed $X R C C 2$ expression in various tumor cell lines (colon cancer cell lines T84, HT29 and Lovo, breast cancer cell line MCF-7, esophageal cancer cell line EC9706) and normal HEK293 cell line. As shown in Figure 1, overexpression of XRCC2 appeared in tumor cell lines compared with HEK293. Given the elevated level of XRCC2 protein in T84 colon cancer cell line, we selected T84 in subsequent experiment.

Figure 1. XRCC2 protein was expressed highly in T84 colon cancer cell line. Total protein of various tumor cell lines (colon cancer cell lines T84, HT29 and Lovo, breast cancer cell line MCF-7, esophageal cancer cell line EC9706) and normal HEK293 cell line were extracted, and the $X R C C 2$ expression was examined by Western blot.

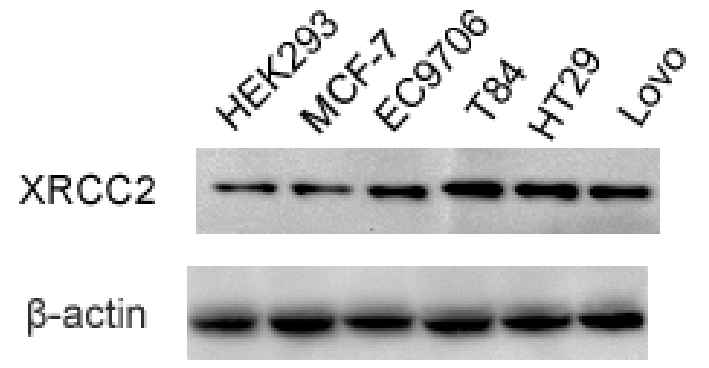

\subsection{Knockdown of XRCC2 Using Vector-Based shRNA in T84 Cells}

RNAi technology was used to knockdown XRCC2 expression in T84 cells. The vector-based shRNA plasmid (shRNA-XRCC2) were transfected into T84 cells. The scrambled cells were treated with control shRNA plasmid-A as scramble shRNA (shRNA-SC). The efficiency of transfection was evaluated by the levels of $X R C C 2$ mRNA and protein expression. XRCC2 expression was lower in 
shRNA-XRCC2 cells than in shRNA-SC cells (Figure 2), indicating that XRCC2 expression was effectively suppressed by shRNA-XRCC2.

Figure 2. XRCC2 expression was suppressed by vector-based shRNA in T84 cells. (A) XRCC2 protein expression; and (B) XRCC2 mRNA expression. Cells were transfected with either shRNA-XRCC2 or shRNA-SC. Total protein and mRNA levels of XRCC2 were determined at $24 \mathrm{~h}$ after transfection by Western blot and quantitative real-time polymerase chain reaction (PCR) analyses, respectively. The values are presented as the mean $\pm \mathrm{SD}(n=6) .{ }^{*} p<0.01$ compared with the control group.

A

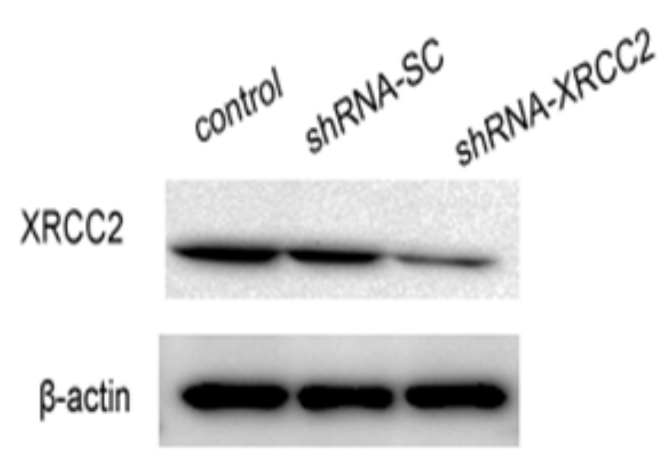

B

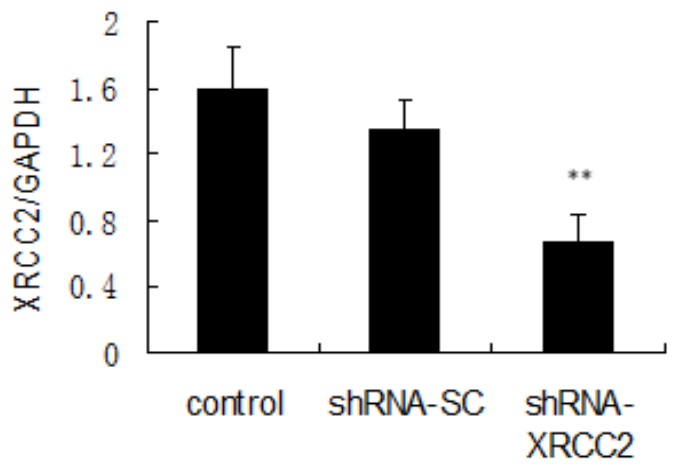

\subsection{Effect of XRCC2 Knockdown on Cell Growth of T84 Cells}

To investigate the role of $X R C C 2$ on the growth of T84 cells, we analyzed the growth curve of cells in vitro by MTT assay. Cells of control group and shRNA-SC group grew steadily by linear growth. An obvious decrease in the cell proliferation rate was found on the third day in the shRNA-XRCC2 cells compared with shRNA-SC transfectant and control cells (Figure 3). Results revealed that shRNA-XRCC2 resulted in more efficient inhibition of cell growth than shRNA-SC.

Figure 3. Knockdown of XRCC2 by shRNA inhibited cell growth of T84 cells. Cells were transfected with either shRNA-XRCC2 or shRNA-SC. The effect of XRCC2 suppression on cell growth in T84 cell line was examined by MTT assay. The values are presented as the mean $\pm \mathrm{SD}(n=12) .{ }^{*} p<0.05,{ }^{* *} p<0.01$ compared with the control group.

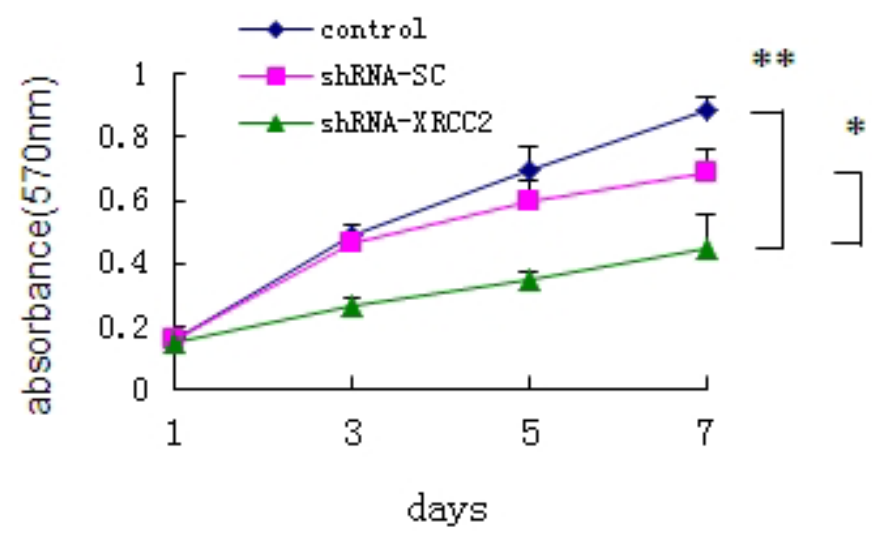




\subsection{Effect of XRCC2 Knockdown on the Response of T84 Cells to X-Radiation}

To determine the effect of $X R C C 2$ knockdown on radiosensitivity to X-radiation of T84 cells, we examined their ability to form colonies in vitro (Figure 4). The number of colonies formed by shRNA-XRCC2 cells was significantly decreased compared with that of control cells. However, there was no significant change in shRNA-SC cells compared with control cells. Compared with shRNA-XRCC2 group and radiation group, the number of colonies in shRNA-XRCC2 combined radiation group was decreased significantly. The results suggested that shRNA-XRCC2 cells were more sensitive to radiation than shRNA-SC and control cells.

Figure 4. Knockdown of $X R C C 2$ by shRNA enhanced radiosensitivity to X-radiation of T84 cells. (A) Photographs of colonies; and (B) the number of colonies. Cells were treated with either shRNA-XRCC2, shRNA-SC, radiation or shRNA-XRCC2 combined radiation. Cells of control group, shRNA-SC group and shRNA-XRCC2 group were not exposed to radiation. Cells of radiation group were treated with 8 Gy X-ray radiation after T84 cells attached. shRNA-XRCC2 combined radiation group received 8 Gy radiation at $12 \mathrm{~h}$ after shRNA-XRCC2 transfection. The effect of XRCC2 suppression on radiosensitivity of T84 cell line was examined by colony formation assay. The values are presented as the mean $\pm \mathrm{SD}(n=6) .{ }^{*} p<0.05, * * p<0.01$ compared with the control group.

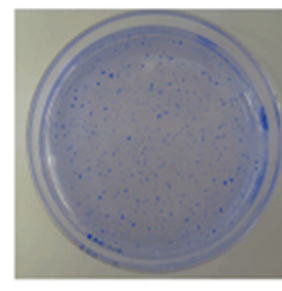

control

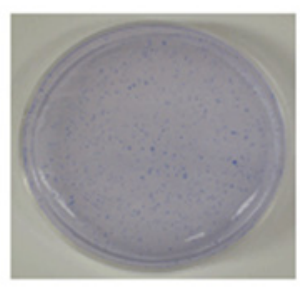

ShRNA-SC

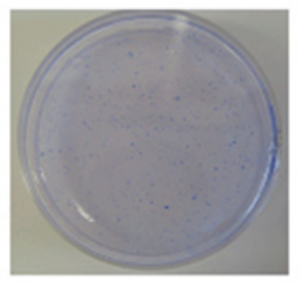

ShRNA-XRCC2

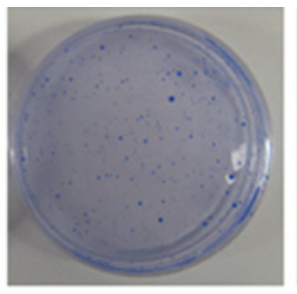

radiation

$\mathbf{A}$
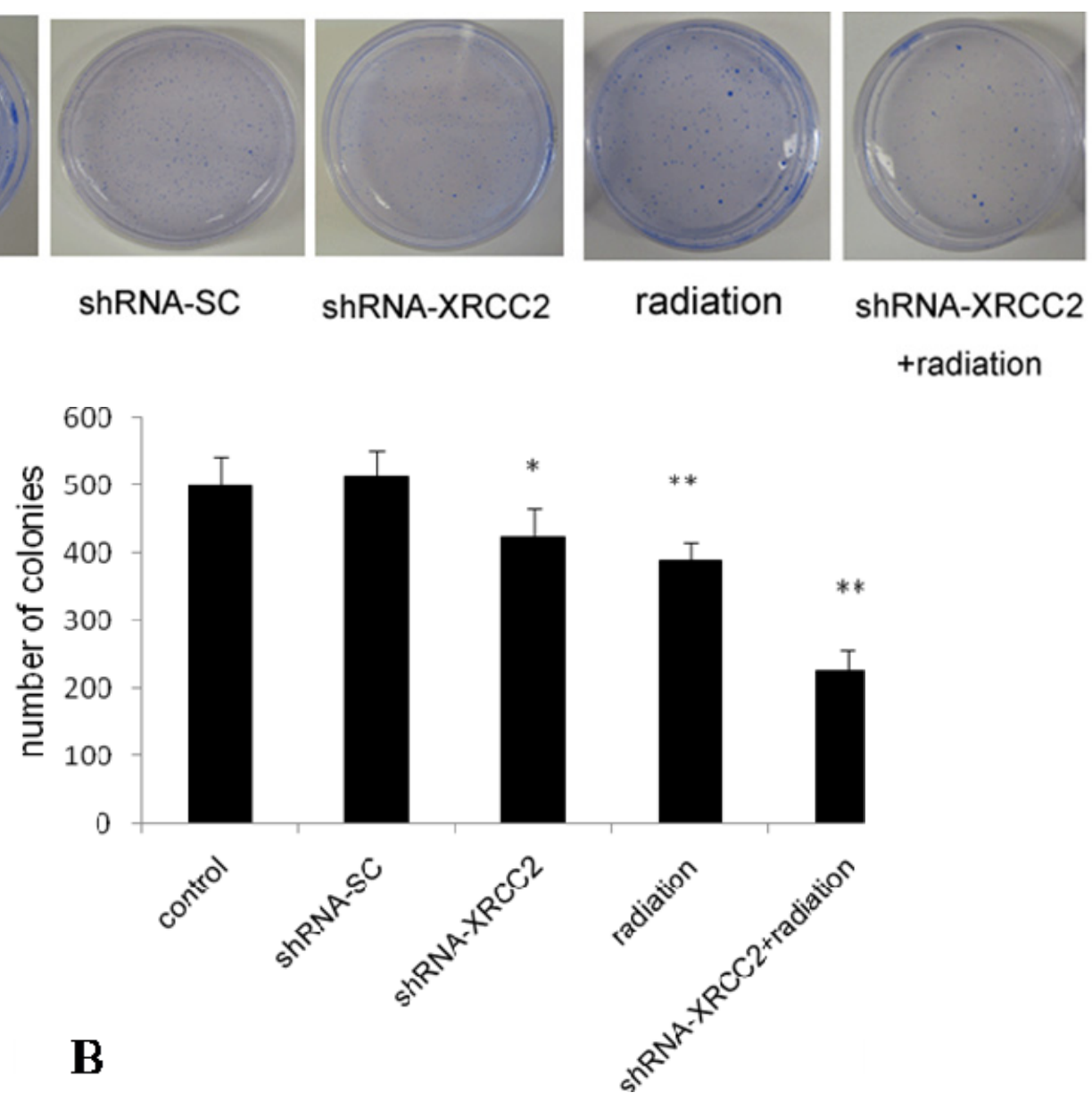


\subsection{Effect of XRCC2 Knockdown on Cell Cycle Distribution Induced by Radiation}

We analyzed the relationship between the increased sensitivity of shRNA-XRCC2 cells to IR with the cell cycle distribution. The proportion of shRNA-XRCC2 cells in the G2/M phase was higher than that of shRNA-SC cells and control cells without radiation treatment. When exposed to 8 Gy radiation, the shRNA-XRCC2 cell population in the G2/M phase was significantly increased, and those in the G1 phase was decreased compared with control cells (Figure 5). The results suggested that the knockdown of XRCC2 enabled XRCC2 tumor cells to escape from G1 phase and to arrest in the G2/M phase.

Figure 5. Knockdown of $X R C C 2$ arrested cells in the G2/M phase. (A) Cell cycle distribution without exposure to radiation; and $(\mathbf{B})$ cell cycle distribution after exposure to 8 Gy radiation. Cells were transfected with either shRNA-XRCC2 or shRNA-SC. Cells were irradiated with 8 Gy at $12 \mathrm{~h}$ after transfection. The effect of $X R C C 2$ suppression on cell cycle distribution in T84 cell line was examined by flow cytometric analysis. The values are presented as the mean $\pm \operatorname{SD}(n=5) . * p<0.05, * * p<0.01$ compared with the control group.

A

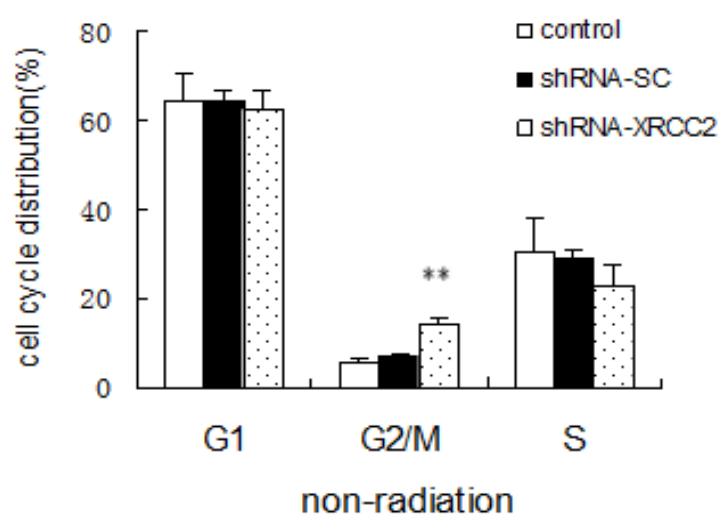

B

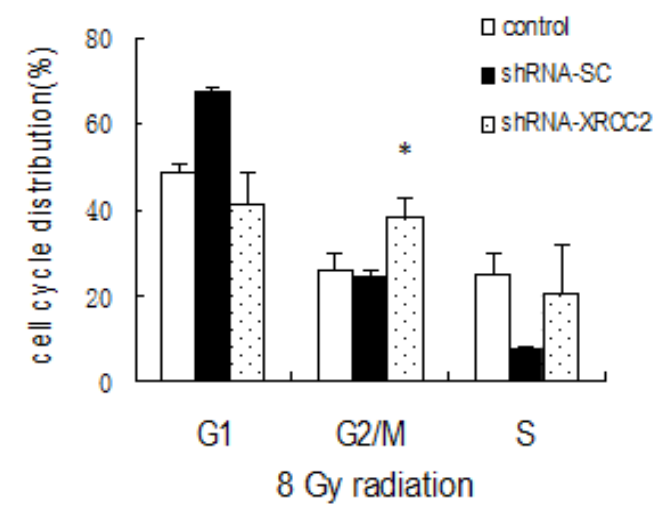

\subsection{Effect of XRCC2 Knockdown on Cell Apoptosis Induced by Radiation}

To investigate whether the increased sensitivity of cells transfected with shRNA-XRCC2 after exposure to radiation is related to apoptosis, we examined cell apoptosis by flow cytometry. The number of apoptotic cells of shRNA-XRCC2 group was higher than that of control and shRNA-SC cells without radiation treatment. When irradiated with 8 Gy radiation, the number of shRNA-XRCC2 apoptotic cells was increased compared with that of control and shRNA-SC cells, which showed that shRNA-XRCC2 cells were more sensitive to apoptosis induced by radiation (Figure 6). These findings suggested that the knockdown of XRCC2 expression mediated radiosensitization of T84 cells, and was associated with enhanced apoptosis. 
Figure 6. Knockdown of $X R C C 2$ enhanced cell apoptosis after radiation treatment. (A) Cell apoptosis without exposure to radiation; and (B) cell apoptosis after exposure to 8 Gy radiation. Cells were transfected with either shRNA-XRCC2 or shRNA-SC. Cells were irradiated with $8 \mathrm{~Gy}$ at $12 \mathrm{~h}$ after transfection. The effect of XRCC2 suppression on cell apoptosis in T84 cell line was examined by flow cytometric analysis. The values are presented as the mean $\pm \operatorname{SD}(n=5)$. $* * p<0.01$ compared with the control group.

A

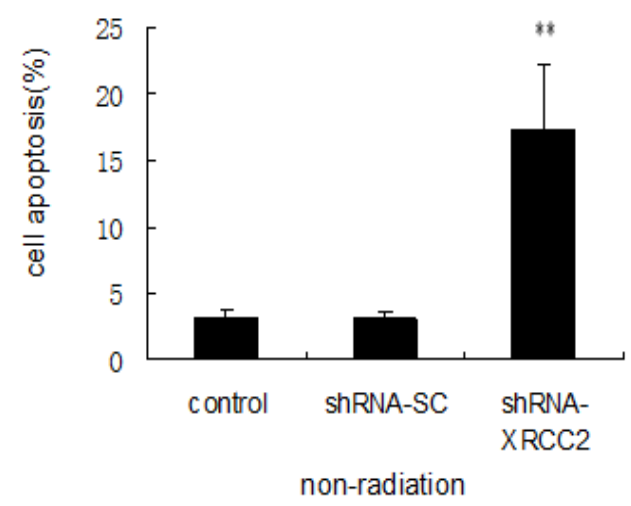

B

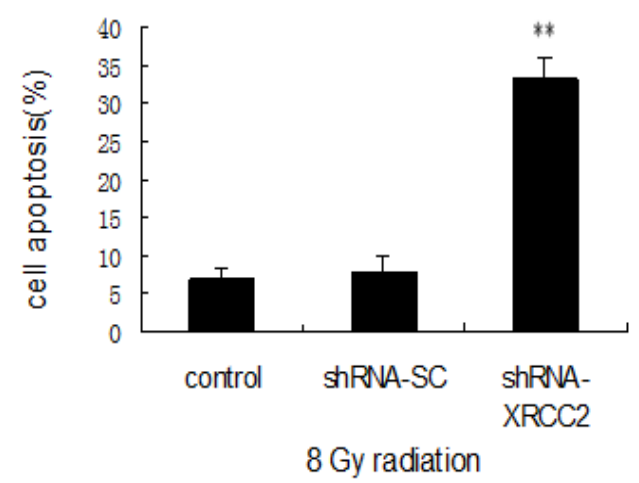

\subsection{Effect of XRCC2 Knockdown on Tumorigenicity in Nude Mice}

To determine the effect of shRNA-XRCC2 on tumor growth in vivo, a xenograft model was performed in nude mice. As shown in Figure 7A, tumors of control group and shRNA-SC group grew abruptly, whereas those of shRNA-XRCC2 group grew steadily. Tumor growth was retarded in shRNA-XRCC2 group compared with control group. At day 28 after exposure to radiation, the tumor volume of shRNA-XRCC2 group was decreased significantly compared with that of control group. In addition, the tumor weight was also lighter in shRNA-XRCC2 group than in control group after mice were sacrificed (Figure 7B). As shown in Figure 7C,D, the tumors of shRNA-XRCC2 transfected cells were obviously smaller than that of shRNA-SC and control cells. The results suggested that the knockdown of $X R C C 2$ rendered colon tumor cells more sensitive to reradiation.

\subsection{Effect of XRCC2 Knockdown on Pathology in Nude Mice}

The mice were sacrificed at 28 days after radiation treatment. Tumors were harvested for pathological analysis. The pathological examination revealed detailed effect of XRCC2 knockdown on tumor growth in vivo (Figure 8). Tumor tissue arrangement showed gland-like structure and no obvious capsule was seen in dissected tumors of three groups. Pathological analysis of control group and shRNA-SC group was essentially identical: some inflammatory cells were seen in glandular cavity and infiltrated to peritumor; karyokinetic figure was commonly seen and no obvious necrosis appeared in tumor tissue. However, small areas of necrosis were found in shRNA-XRCC2 group (Table 1). Our data suggested that treatment of shRNA-XRCC2 transfection had better anti-tumor effect than that of control and shRNA-SC. 
Figure 7. Knockdown of $X R C C 2$ decreased tumor growth in nude mice. (A) Tumor growth curve; (B) Tumor weight after mice were sacrificed; (C) Ectopic T84 xenografts in nude mice; and (D) Photograph of the dissected tumors. The effect of shRNA-XRCC2 on tumorigenicity was examined in nude mice. Mice were treated with either shRNA-XRCC2 or shRNA-SC. Radiation treatment ( 2 Gy) was given once every other day when tumor volume reached $100 \mathrm{~mm}^{3}$ in a total of 10 days. The tumor volume and tumor weight was examined for 28 days after exposure to radiation. The values are presented as the mean $\pm \mathrm{SD}$ $(n=10) .{ }^{*} p<0.01$ compared with the control group.

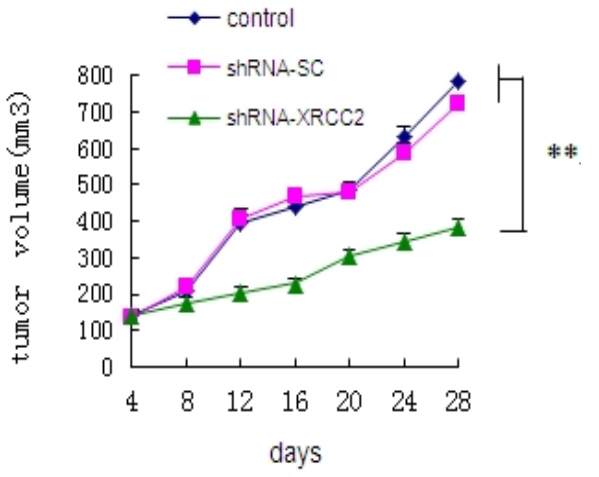

(A)

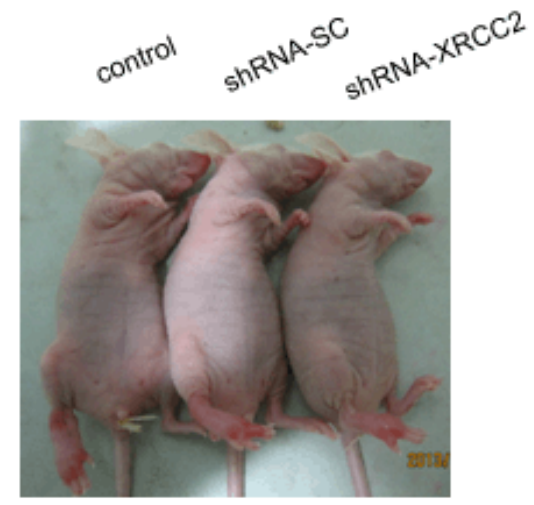

(C)

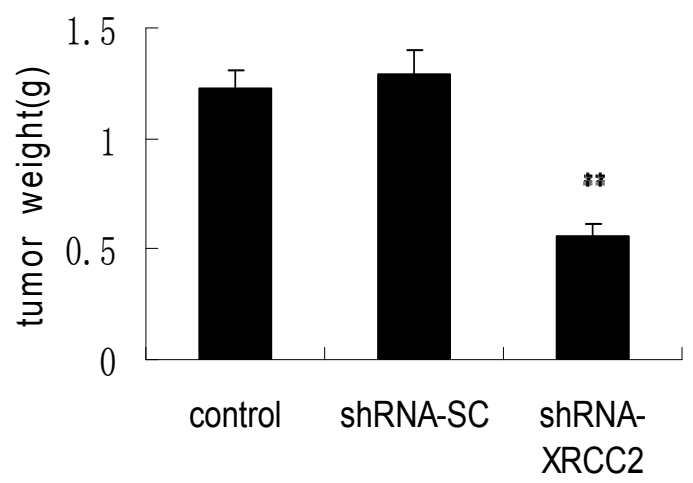

(B)

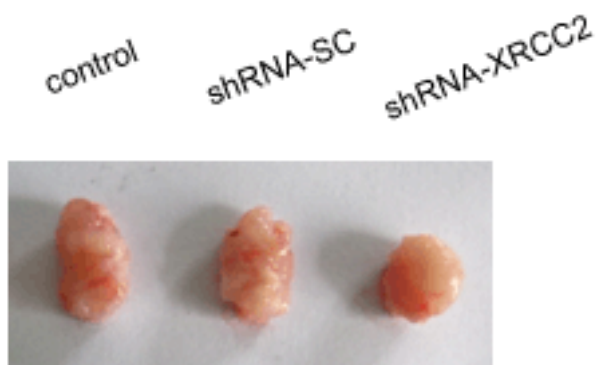

(D)

Figure 8. Pathological analysis of knockdown of $X R C C 2$ expression. The effect of XRCC2 shRNA on pathology was examined in nude mice. Mice were treated with either shRNA-XRCC2 or shRNA-SC. Radiation treatment ( 2 Gy) was given once every other day when tumor volume reached $100 \mathrm{~mm}^{3}$ in a total of 10 days. The tumor pathology was examined on day 28 after exposure to radiation. (Magnification: $\times 400$; Scale bar: $100 \mu \mathrm{m}$ ).

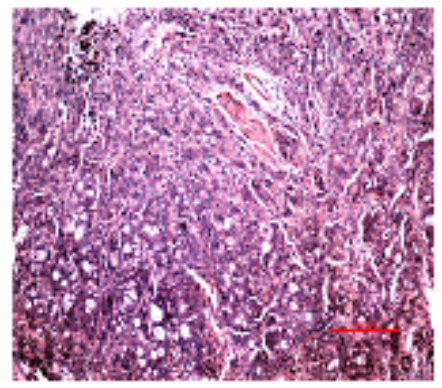

control

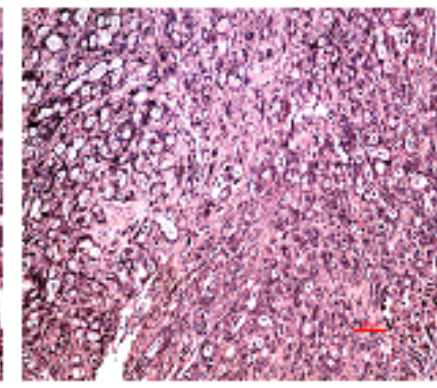

shRNA-SC

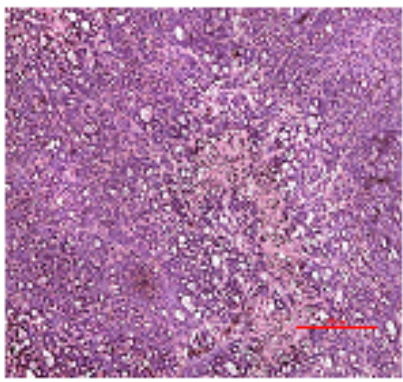

shRNA-XRCC2 
Table 1. Pathological analysis of harvested tumors in nude mice.

\begin{tabular}{cccc}
\hline & Control & shRNA-SC & shRNA-XRCC2 \\
\hline Tumor margin & + & + & + \\
Architectural atypia & + & + & $+/-$ \\
Cellular atypia & + & + & + \\
Karyokinesis & ++ & ++ & + \\
Necrotic area & $+/-$ & $+/-$ & + \\
\hline
\end{tabular}

\section{Discussion}

The resistance to radiation therapy is a critical element of affecting the survival of colon cancer patients, and is attributable to various mechanisms [20,21]. The role of the DNA repair protein XRCC2 on colon cancer radioresistance remains poorly understood. In the study, we adopted vector-based shRNA expression system to investigate the effect of XRCC2 silencing on sensitization to X-radiation in colon cancer in vitro and in vivo.

A recent study showed that the incidence of spontaneous breast and intestinal tumorigenesis was higher in $\mathrm{XRCC}^{+/+}$mice than in $\mathrm{XRCC}^{+/-}$animals [22], implicating a possible role for XRCC2 in affecting tumor cells' radiosensitivity. In the present study, we found that the expression of XRCC2 protein was elevated in colon cancer cells compared with normal cells. As uncontrolled growth is one of important alteration in cancer cell phenotypes, we firstly examined the effect of XRCC2 knockdown on the growth of T84 colon tumor cells. Our experimental data indicated that the suppression of $X R C C 2$ expression effectively decreased cellular proliferation in vitro. In addition, tumor xenograft studies showed XRCC2 silencing similarly inhibited tumor growth in vivo, suggesting XRCC2 as an important regulator of colon tumor cellular proliferation.

To test this possibility, we studied the effect of $X R C C 2$ suppression on radiosensitivity of colon cancer cells. We found that shRNA-mediated XRCC2 suppression rendered T84 tumor cells more sensitive to radiation treatment as evaluated by the colony formation assay. The XRCC2-mediated enhancement of radiosensitivity was associated with an elevation of radiation-induced apoptosis, suggesting a possible role of XRCC2 in the regulation of radiation-induced apoptosis. Our findings were consistent with previous reports that silencing XRCC2 expression using RNA interference technology similarly improved the radiosensitivity of human brain tumor and lung cancer cells [23]. In addition, our investigation showed that lowering XRCC2 expression level enhanced the sensitivity of tumors to radiation in nude mice. Collectively, our experimental data suggest that knockdown of $X R C C 2$ expression enhances colon tumor cells' sensitivity to radiotherapy in vitro and in vivo.

The cell cycle phase is one of the most important determinant of radiosensitivity, with cells being most radiosensitive in the $\mathrm{G} 2 / \mathrm{M}$ phase, less sensitive in the G1 phase, and least sensitive during the latter part of the S phase. Our results showed that cells transfected with shRNA-XRCC2 were arrested in $\mathrm{G} 2 / \mathrm{M}$ phase after exposed to radiation. As cells arrested in the $\mathrm{G} 2 / \mathrm{M}$ phase are generally more sensitive to radiation than those in other phases [24], it is plausible that the XRCC2-mediated change of the cell cycle may consequently affect the sensitivity of tumor cells to radiation therapy.

Although several reports have suggested an association between XRCC2 expression and radiosensitivity [8,9], it is unclear whether suppression of $X R C C 2$ expression in any tumor cells uniformly facilitates tumor radiotherapy. Similarly, the molecular mechanisms by which XRCC2 
mediates tumor cells' radiosensitivity remains a subject of future investigation. Given that inhibiting of $X R C C 2$ expression can enhance radiosensitivity of tumor cells, strategies to knockdown XRCC2 expression may improve the efficacy of radiation therapy in clinical settings.

In summary, knockdown of XRCC2 significantly sensitized colon tumor cells to radiation, as assayed in both in vitro cell culture and in vivo xenograft tumor models. The study suggest that XRCC2 might be a radiotherapy target in colon cancer, and that XRCC2 gene-specific therapy may be developed as an assistant method of enhancing the antitumor effect of traditional radiation treatment.

\section{Experimental Section}

\subsection{Cell Culture}

The human colon cancer cell line (T84, HT29 and Lovo) was purchased from the Cell Culture Center of Basic Medicine, Chinese Academy of Medical Sciences, Beijing, China. Breast cancer cell line MCF-7, esophageal cancer cell line EC9706 and normal human embryonic kidney cell line HEK293 were obtained from our laboratory. Cells were cultured in DMEM/F12 (Hyclone, Beijing, China) supplemented with $10 \%$ FBS and $100 \mu \mathrm{g} / \mathrm{mL}$ of penicillin/streptomycin, in a humidified $5 \%$ $\mathrm{CO}_{2}$ incubator at $37^{\circ} \mathrm{C}$.

\subsection{Ionizing Radiation}

Cells and mice were exposed to ionizing radiation (IR) in a X irradiator (Rad source Inc., Suwanee, GA, USA) at a rate of $0.99 \mathrm{~Gy} / \mathrm{min}$.

\section{3. shRNA Plasmid Stable Transfection}

T84 cells were stably transfected with either shRNA XRCC2 plasmid (sc-36861-SH, Santa Cruz, Dallas, TX, USA) (shRNA-XRCC2) or control shRNA plasmid-A (sc-108060, Santa Cruz) as scramble shRNA (shRNA-SC), using Lipofectin reagent (Invitrogen, Carlsbad, CA, USA), according to the manufacturer's instruction. Briefly, $1 \mu \mathrm{g}$ of each plasmid DNA and $20 \mu \mathrm{L}$ of Lipofectin 2000 (Invitrogen) were mixed separately with serum-free Optim-MEM medium (Invitrogen) and incubated for $5 \mathrm{~min}$ at room temperature. Then they were combined and incubated for $15 \mathrm{~min}$. After $48 \mathrm{~h}$ incubation, individual colonies were selected for resistance to $10 \mu \mathrm{g} / \mathrm{mL}$ puromycin for 2 weeks. Signal colonies were isolated and cultured to obtain stably transfected cells line. The effectiveness of transfection was examined by Western blot.

\subsection{MTT Assay}

T84 cells were planted at a density of 1000/well in a 96-well plate in triplicate. After cells adhered, samples were infected with either shRNA-XRCC2 or shRNA-SC and cultured at $37^{\circ} \mathrm{C}$. Each well was added $20 \mu \mathrm{L}$ MTT (5 $\mu \mathrm{g} / \mathrm{mL}$, Amresco Inc., Solon, OH, USA) on day 1, 3, 5, and 7, and incubated for $4 \mathrm{~h}$. The medium was removed and $150 \mu \mathrm{L}$ dimethyl sulphoxide (DMSO) was added. The absorbance of each well was measured by the Multi-Mode Microplate Reader (Synergy HT, BioTek, Winooski, VT, USA) at $570 \mathrm{~nm}$. 


\subsection{Colony Formation Assay}

A colony formation assay was performed to determine sensitivity of T84 cells to X-radiation. Eight hundred T84 cells were seeded in $55 \mathrm{~mm}$ dishes and allowed to attach. Cells were treated with either shRNA-XRCC2, shRNA-SC, radiation, or shRNA-XRCC2 combined radiation. The control group did not receive any treatment. Radiation group were treated with 8 Gy X-ray radiation after T84 cells attached. ShRNA-XRCC2 combined radiation group received 8 Gy radiation at $12 \mathrm{~h}$ after shRNA-XRCC2 transfection. Colonies were fixed by methanol and stained with the Giemsa stain at day 14 after incubation. A minimum of 50 viable cells were scored as a colony.

\subsection{Western Blot Analysis}

Cells $\left(1 \times 10^{6}\right.$ cells $)$ were seeded in $100 \mathrm{~mm}$ dishes. After cells adhered, samples were infected with either shRNA-XRCC2 or shRNA-SC. Cells were lysed in mammalian protein extraction reagent (M-PER) (Thermo, Waltham, MA, USA). Total protein concentration was determined using a Bicinchoninic acid (BCA) protein assay kit (Beyotime Biotechnology, Shanghai, China). Total protein $(30 \mu \mathrm{g})$ was loaded onto $10 \%$ gradient SDS-PAGE gels and then transferred to polyvinylidene fluoride (PVDF) membranes (Millipore, Billerica, MA, USA). Membranes were blocked and incubated with the following antibodies: The mouse anti-XRCC2 antibody $(1: 1000$ sc-73278) from Santa Cruz Biotechnology Incorporation, rabbit anti- $\beta$-actin (1:5000, P30002) and goat anti-mouse IgG conjugated to horseradish peroxidase (M21001) from Abmart Incorporation (Beijing, China). Chemiluminescent signal detection was performed by an electrochemical luminescence (ECL) kit (Boster Biotechnology, Abingdon, UK).

\subsection{Quantitative Real-Time PCR}

Cells $\left(1 \times 10^{6}\right.$ cells $)$ were seeded in $100 \mathrm{~mm}$ dishes. After cells adhered, samples were infected with either shRNA-XRCC2 or shRNA-SC. XRCC2 mRNA expression was performed by Quantitative real-time PCR (qPCR). Total RNA was prepared using Trizol reagent (Invitrogen) according to the manufacturer's instruction. The first strand of cDNA was synthesized using RNA PCR kit (Takara Bio Inc., Dalian, China). qPCR was performed on the CFX96 (BioRad, Hercules, CA, USA) with SYBR Green kit (Takara Bio Inc.) according to the manufacturer's instruction. The sequences of the primers were as follows: XRCC2 forward 5'-CAATGGAGGAGAAAGTGTGAACT-3', XRCC2 reverse 5'-CAAAAAGAACCAGGCGATAGTC-3', GAPDH (set as the reference) forward 5'-CGAATTGGCTACAGCAACAGG-3', GAPDH reverse 5'-GTACATGACAAGGTGCGGCTC-3'. The primers were obtained from Sangon Biotech (Shanghai, China).

\subsection{Cell Cycle}

Cell cycle distribution was measured by flow cytometric analysis. Cells $\left(1 \times 10^{5}\right.$ cells/well $)$ were seeded in 6-well culture plates. After cells adhered, samples were infected with either shRNA-XRCC2 or shRNA-SC. Radiation treatment was given $8 \mathrm{~Gy}$ X-radiation at $12 \mathrm{~h}$ after transfection and then further cultured for $24 \mathrm{~h}$. Cells were harvested by trypsinization and fixed in ice-cold $70 \%$ ethanol overnight. After washing with PBS, the fixed cells were stained with propidium iodide solution 
( $50 \mu \mathrm{g} / \mathrm{mL}$ propidium iodide, $0.1 \%$ Triton $\mathrm{X}-100$, and $0.1 \%$ sodium citrate in PBS) for $30 \mathrm{~min}$ at $4{ }^{\circ} \mathrm{C}$ in the dark. The DNA content was determined by a FACS cytometer (Becton-Dickinson, Franklin Lakes, NJ, USA) using CellQuest program (Becton-Dickinson) and data from $\geq 10,000$ cells were analyzed with ModFit software (Becton-Dickinson).

\subsection{Cell Apoptosis}

Cells apoptosis were determined with FITC annexin V apoptosis detection kit (556570, BD pharmingen, Taipei, Taiwan) using flow cytometric analysis. Cells $\left(1 \times 10^{5}\right.$ cells/well $)$ were seeded in 6-well culture plates. After cells adhered, samples were infected with either shRNA-XRCC2 or shRNA-SC. Radiation treatment was given 8 Gy X-radiation at $12 \mathrm{~h}$ after transfection and then further cultured for $24 \mathrm{~h}$. Cells were harvested by trypsinization and incubated in $0.5 \mathrm{~mL}$ of binding buffer containing $0.5 \mu \mathrm{g} / \mathrm{mL}$ Annexin-V-FITC and $5 \mu \mathrm{g} / \mathrm{mL}$ propidium iodide for $30 \mathrm{~min}$ in the dark. Cells apoptosis was measured by a FACS cytometer (Becton-Dickinson) using the CellQuest program and data from $\geq 10,000$ cells were analyzed with ModFit software.

\subsection{Tumorigenicity in Nude Mice}

Six-week-old male BALB/c nude mice were purchased from Academy Military Medical Sciences (Beijing, China). The experimental protocol was approved by the China Institutional Ethics Review Committee for Animal Experimentation. Cells $\left(1 \times 10^{7} /\right.$ mouse $)$ suspended in $0.2 \mathrm{~mL}$ DMEM/F12 medium were injected subcutaneously into the right leg of nude mice. The mice were randomly divided into three groups: control, shRNA-SC, and shRNA-XRCC2, five mice in each group. Radiation treatment ( 2 Gy) was given once every other day when tumor volume reached $100 \mathrm{~mm}^{3}$ in a total of 10 days. The tumor volume was measured over the skin from an external caliper using the formula: $\mathrm{V}\left(\mathrm{mm}^{3}\right)=1 / 6 \pi \times$ length $(\mathrm{mm}) \times \operatorname{width}^{2}\left(\mathrm{~mm}^{2}\right)$. The nude mice were anaesthetized using chloral hydrate solution $(0.3 \mathrm{~g} / \mathrm{kg})$ intraperitoneally before radiation was delivered to the tumor area with the rest of the mice shielded. The mice were sacrificed on the twenty eighth day after radiation treatment. Tumors were dissected, weighed, and harvested for pathological analysis.

\subsection{Pathological Analysis}

Dissected tumors were fixed in $4 \%$ neutral buffered formalin and embedded in paraffin using standard procedures. Paraffin embedded tumors were cut in $5 \mu \mathrm{m}$ sections at the maximum cross-section. Sections were stained with haematoxylineosin (H.E.) and analyzed under a microscope (BX51, Olympus, Shinjuku, Japan) at 400-fold magnification.

\subsection{Statistical Analysis}

All experiments were repeated at least two times. Data are presented as mean $\pm \mathrm{SD}$. Differences between groups were calculated using the Student's $t$ test. $p$-values $<0.05$ were considered as statistically significant. 


\section{Conclusions}

In conclusion, knockdown of XRCC2 expression has radiosensitization effects on colon tumor cells in vitro and in vivo. These data strongly suggested that XRCC2 may be further developed as a promising therapeutic target for the treatment of radioresistant human colon cancer.

\section{Acknowledgments}

We thank Haichao Wang (The Feinstein Institute for Medical Research, Manhasset, NY 11030, USA) for critical reading of the revised manuscript. This study was supported by the Special Foundation of the Ministry of Health (No. 201002009); National Natural Science Foundation of China (No.30870583, 31170804, 31240052, 31200634, and 31300695); the Natural Science Foundation of Tianjin (No. 09JCYBJC09300, 11ZCGYSY02400, 12JCYBJC15300, 12JCYBJC32900, 13JCYBJC23500, and 13JCQNJC11600); the Science Research Foundation for Doctor-Subject of High School of the National Education Department (No. 20101106110046, 20121106120044, and 20121106120043); and the PUMC Youth Fund and Fundamental Research Funds for the Central Universities (No. 2012G01, and 2012J05).

\section{Author Contributions}

The work presented here was carried out in collaboration between all authors. Qin Wang, Feiyue Fan and Qiang Liu conceived and designed the experiments. Qin Wang, Yan Wang and Liqing Du carried out the laboratory experiments, analyzed the data, interpreted the results and wrote the paper. Chang Xu, Yue Fu and Yuanming Sun co-worked on associated data collection and their interpretation. Bing Yang and Lu Cai contributed significantly to analysis and manuscript preparation. Zhijuan Sun and Saijun Fan helped perform the analysis with constructive discussions. All authors have contributed to, seen and approved the manuscript.

\section{Conflicts of Interest}

The authors declare no conflict of interest.

\section{References}

1. Jemal, A.; Bray, F.; Center, M.M.; Ferlay, J.; Ward, E.; Forman, D. Global cancer statistics. CA Cancer J. Clin. 2011, 61, 69-90.

2. Center, M.M.; Jemal, A.; Smith, R.A.; Ward, E. Worldwide variations in colorectal cancer. CA Cancer J. Clin. 2009, 59, 366-378.

3. Ganapathi, S.; Kumar, D.; Katsoulas, N.; Melville, D.; Hodgson, S.; Finlayson, C.; Hagger, R. Colorectal cancer in the young: Trends, charactistics and outcome. Int. J. Color. Dis. 2011, 26, 927-934.

4. Tambini, C.E.; Spink, K.G.; Ross, C.J.; Hill, M.A.; Thacker, J. The importance of XRCC2 in RAD51-related DNA damage repair. DNA Repair 2010, 9, 517-525. 
5. Romanowicz, H.; Smolarz, B.; Baszczyński, J.; Zadrożny, M.; Kulig, A. Genetics polymorphism in DNA repair genes by base excision repair pathway (XRCC1) and homologous recombination (XRCC2 and RAD51) and the risk of breast carcinoma in the Polish population. Pol. J. Pathol. 2010, 61, 206-212.

6. Rajesh, C.; Gruver, A.M.; Basrur, V.; Pittman, D.L. The interaction profile of homologous recombination repair proteins RAD51C, RAD51D and XRCC2 as determined by proteomic analysis. Proteomics 2009, 9, 4071-4086.

7. Park, S.W.; Yoo, N.J.; Lee, S.H. Mutational analysis of mononucleotide repeats in XRCC2 and XRCC6 in cancers with microsatellite instability. Pathology 2011, 43, 78-79.

8. Liu, Y.; Shete, S.; Wang, L.E.; El-Zein, R.; Etzel, C.J.; Liang, F.W.; Armstrong, G.; Tsavachidis, S.; Gilbert, M.R.; Aldape, K.D.; et al. $\gamma$-Radiation sensitivity and polymorphisms in RAD51L1 modulate glioma risk. Carcinogenesis 2010, 31, 1762-1769.

9. Shi, W.L.; Li, J.; Chen, P.; Dai, C.H. Effect of X-ray on expression levels of XRCC2 and XRCC3 in lung cancer cell line A549. Acta Univ. Med. Nanjing Nat. Sci. 2009, 29, 1517-1520.

10. Lin, W.Y.; Camp, N.J.; Cannon-Albright, L.A.; Allen-Brady, K.; Balasubramanian, S.; Reed, M.W.; Hopper, J.L.; Apicella, C.; Giles, G.G.; Southey, M.C.; et al. A role for XRCC2 gene polymorphisms in breast cancer risk and survival. Med. Genet. 2011, 48, 477-484.

11. Shamseldin, H.E.; Elfaki, M.; Alkuraya, F.S. Exome sequencing reveals a novel Fanconi group defined by XRCC2 mutation. J. Med. Genet. 2012, 49, 184-186.

12. Silva, S.N.; Tomar, M.; Paulo, C.; Gomes, B.C.; Azevedo, A.P.; Teixeira, V.; Pina, J.E.; Rueff, J.; Gaspar, J.F. Breast cancer risk and common single nucleotide polymorphisms in homologous recombination DNA repair pathway genes XRCC2, XRCC3, NBS1 and RAD51. Cancer Epidemiol. 2010, 34, 85-92.

13. Bastos, H.N.; Antao, M.R.; Silva, S.N.; Azevedo, A.P.; Manita, I.; Teixeira, V.; Pina, J.E.; Gil, O.M.; Ferreira, T.C.; Limbert, E.; et al. Association of polymorphisms in genes of the homologous recombination DNA repair pathway and thyroid cancer risk. Thyroid 2009, 19, 1067-1075.

14. Pearce, C.L.; Near, A.M.; van den Berg, D.J.; Ramus, S.J.; Gentry-Maharaj, A.; Menon, U.; Gayther, S.A.; Anderson, A.R.; Edlund, C.K.; Wu, A.H.; et al. Validating genetic risk associations for ovarian cancer through the international ovarian cancer association consortium. Br. J.Cancer 2009, 100, 412-420.

15. Krupa, R.; Sliwinski, T.; Wisniewska-Jarosinska, M.; Chojnacki, J.; Wasylecka, M.; Dziki, L.; Morawiec, J.; Blasiak, J. Polymorphisms in RAD51, XRCC2 and XRCC3 genes of the homologous recombination repair in colorectal cancer-A case control study. Mol. Biol. Rep. 2011, 38, 2849-2854.

16. Slattery, M.L.; Curtin, K.; Wolff, R.K.; Boucher, K.M.; Sweeney, C.; Edwards, S.; Caan, B.J.; Samowitz, W. A comparison of colon and rectal somatic DNA alterations. Dis. Colon Rectum 2009, 52, 1304-1311.

17. Curtin, K.; Lin, W.Y.; George, R.; Katory, M.; Shorto, J.; Cannon-Albright, L.A.; Bishop, D.T.; Cox, A.; Camp, N.J.; Colorectal Cancer Study Group. Meta association of colorectal cancer confirms risk alleles at 8q24 and 18q21. Cancer Epidemiol. Biomark. Prev. 2009, 18, 616-621. 
18. Curtin, K.; Lin, W.Y.; George, R.; Katory, M.; Shorto, J.; Cannon-Albright, L.A.; Smith, G.; Bishop, D.T.; Cox, A.; Camp, N.J.; et al. Genetic variants in XRCC2: New insights into colorectal cancer tumorigenesis. Cancer Epidemiol. Biomark. Prev. 2009, 18, 2476-2484.

19. Reeves, S.G.; Meldrum, C.; Groombridge, C.; Spigelman, A.; Suchy, J.; Kurzawski, G.; Lubinski, J.; Scott, R.J. DNA repair gene polymorphisms and risk of early onset colorectal cancer in Lynch syndrome. Cancer Epidemiol. 2012, 36, 183-189.

20. Gladstone, M.; Su, T.T. Radiation responses and resistance. Int. Rev. Cell Mol. Biol. 2012, 299, 235-253.

21. Bai, J.; Guo, X.G.; Bai, X.P. Epidermal growth factor receptor-related DNA repair and radiation-resistance regulatory mechanisms: A mini-review. Asian Pac. J. Cancer Prev. 2012, 13, 4879-4881.

22. Haines, J.W.; Coster, M.R.; Adam, J.; Cheeseman, M.; Ainsbury, E.A.; Thacker, J.; Bouffler, S.D. $\mathrm{Xrcc} 2$ modulates spontaneous and radiation-induced tumorigenesis in $\mathrm{Apc}^{\mathrm{min} /+}$ mice. Mol. Cancer Res. 2010, 8, 1227-1233.

23. Zheng, Z.; Ng, W.L.; Zhang, X.; Olson, J.J.; Hao, C.; Curran, W.J.; Wang, Y. RNAi-mediated targeting of noncoding and coding sequences in DNA repair gene messages efficiently radiosensitizes human tumor cells. Cancer Res. 2012, 72, 1221-1228.

24. Pawlik, T.M.; Keyomarsi, K. Role of cell cycle in mediating sensitivity to radiotherapy. Int. J. Radiat. Oncol. Biol. Phys. 2004, 59, 928-942.

(C) 2014 by the authors; licensee MDPI, Basel, Switzerland. This article is an open access article distributed under the terms and conditions of the Creative Commons Attribution license (http://creativecommons.org/licenses/by/3.0/). 